\title{
Adjustable Tooling System for Air Bending
}

\author{
Joost R. Duflou, a , Hans Vanhove ${ }^{1, b}$ and Willem De Coninck ${ }^{1}$ \\ ${ }^{1}$ Katholieke Universiteit Leuven, Department of mechanical engineering, Belgium \\ ajoost.duflou@mech.kuleuven.be, ${ }^{b}$ hans.vanhove@mech.kuleuven.be
}

\section{Keywords: Sheet Metal Bending, Flexible Tooling, Press Brake Technology}

\begin{abstract}
The productivity of bending on press brakes is strongly affected by the changeover time between consecutive tooling setups. The need for flexible tooling systems that can reduce the setup time is therefore obvious. This is a fortiori true for robot supported bending operations, where the tool system should preferably allow fully automated setup of bending stations.

In this article an innovative tooling system concept is presented that can adjust to different punch profiles (from straight tools to deep gooseneck profiles), provide horn tool functionality and allow setups with open punches for collision avoidance during bending. The Leuven bend tooling design can accommodate fully automated changeover between bending jobs. A prototype version of the Leuven bending tool system has been manufactured and extensively tested. Validation results with respect to achievable load levels and stiffness are reported in the article.
\end{abstract}

\section{Introduction}

Increasing demand for small batch production in sheet metal is leading to a need for more flexible production processes that can handle a wide variety of products.

Sheet metal bending, as performed on a press brake, is a widely used forming process for making linear bends by use of a punch and die combination. Many structural components and lining panels are made by means of this process. Each bend line is created by forcing a sheet into a die, using a punch. In the air bending variant of the process, multiple part angles can be obtained with the same toolset by controlling the punch displacement. However, for parts containing multiple bend lines, often different punch and die combinations are needed to avoid part-tool interference. Frequent tool changes between different bending operations are labor intensive and time consuming tasks resulting in reduced productivity[1,2].

Minimizing the numerous tool changes has been the focus of different studies emphasizing process planning. The optimal choice of toolsets and bending sequence for a single part or for a series of different parts has proven to greatly reduce the number of changeovers [3,4,5]. An optimized layout of the toolsets on the press brake maximizes the use of the available work area and minimizes the handling of the workpiece between bends, thereby reducing the production cost [6].

Hardware strategies like an automated tool library or robotic arm to change tools can reduce the labor intensity, but only have a limited influence on the setup times.

This article focuses on an innovative tooling concept to tackle the challenge of bending different geometries with only a minimal need for tool manipulations. An adaptive punch geometry renders tool changes obsolete and results in an adjustable tool geometry for bending different geometrical details.

The two main properties any bending tool must have are the structural strength and stiffness to withstand the forming forces along with the ability to bend the part without tool/part interference.

Mostly, tools are changed to prevent collision between the sheet metal part and punch or die. Collisions can occur before, during and after the bending operation. A collision before the bending action prevents the correct positioning of the bend line centered on the V-die. A typical example is a hidden corner and is illustrated in Figure 1. 


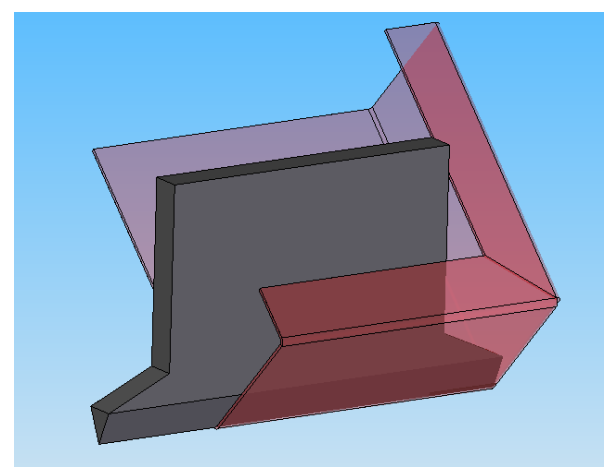

Figure 1. The necessity of a horn tool in hidden corners

During the bending process the sheet metal flanges on both sides of the bend line swivel towards the punch, making this stage collision prone. Therefore well-chosen punch geometries are often required to avoid interference during this step. Different tools with specific geometries are used to avoid collisions, but also collision avoiding bend sequencing strategies are used for parts with multiple bend lines [7]. A typical example for specific tool geometry is the use of a gooseneck punch. This type of punch is used when bending so-called U-details with large flanges.

Collision after the bending process prevents the separation of part and tool. Therefore, dedicated tools with movable parts are sometimes needed.

\section{Concept}

To reduce the time for changing tools, an adjustable punch was developed. This tool is clamped on the press brake and changes geometry when needed, without removing or adding any part. The punch consists of a stationary tip and clamping part (see Fig.2). Multiple C-segments, shown as the C-curved red sections in Figure 2, can rotate around their vertical axis and form the body of the punch.
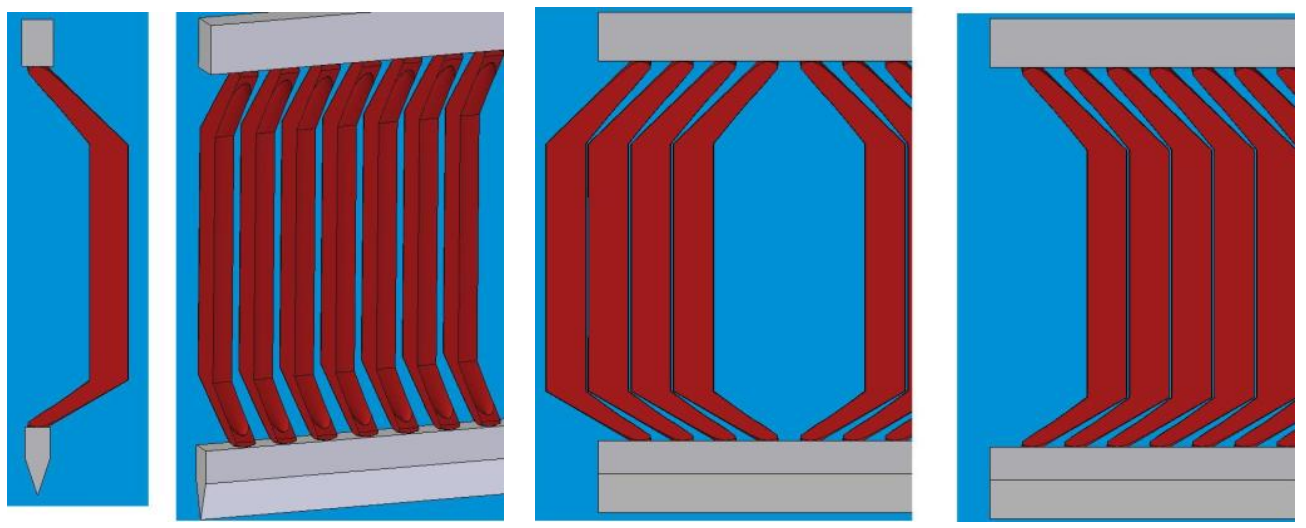

Figure 2. Conceptual representation of the Leuven bend tooling design

The underlying principle for this configuration is the well-chosen positioning of the hinge locations along the center line of the punch in cross sectional view. In this way the moment at the hinge location is always nil in the case of an optimally aligned tool set (Fig. 3). Since the moment is proportional to the axial load, a limited misalignment can be compensated by the high compressive force on the tool, resulting in compressive stress only over the hinge contact area during bending. It can easily be proven that for eccentric loading with eccentricity smaller than $h / 6$ for $h$ the width of the punch tool in the hinge area, positive pressure can be maintained. In the unloaded state the hinges can rotate virtually friction free since there is no force on the contact area between the fixed and rotating parts. 


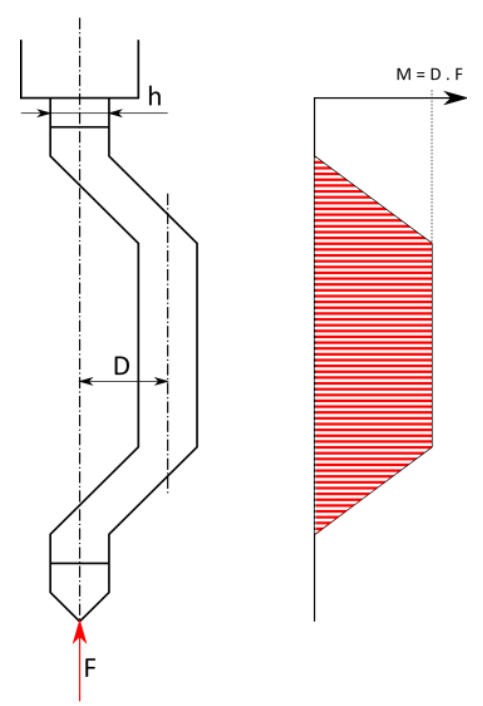

Figure 3 Momentum scheme along the punch under influence of axial loading.

By rotating the $\mathrm{C}$-curved segments, the cross section of the punch can be modified in a continuous way: from a deep gooseneck tool over a straight tool to a deep gooseneck with 180 degree rotated orientation. In its straight cross section orientation, the tool can form a left horn, a right horn, and it is even possible to obtain a hole through the punch for protruding flanges, as illustrated in Figure 2.

\section{Design details}

The $\mathrm{C}$ curved segments were designed to comply with a wide variety of test parts while still being strong enough to resist relatively high bend loads. The C-curved segments were validated on a set of 32 randomly chosen parts provided by Belgian industrial companies. The total set of 32 parts consisted of 188 bend lines, among which there were 85 U-details, 45 Z-details and 21 hidden corners. $99 \%$ of all bend lines in this set were producible with the Leuven bend tooling system.

To automate the rotation of the tool segments, the segments can be driven from the top (just below the clamping zone) using two worm drive systems. This system consists of two worms which can rotate independently. This allows turning of the C-segments connected to the first worm over a different angle than the segments connected to the second worm. By clamping the segments and rotating the worms, the segments stay stationary but the double worm system progresses along the punch (Z-axis) to reposition the separation between the worms. By iterating the turning of the Csegments, every time followed by progressing the worms, for each segment a unique orientation can be set.

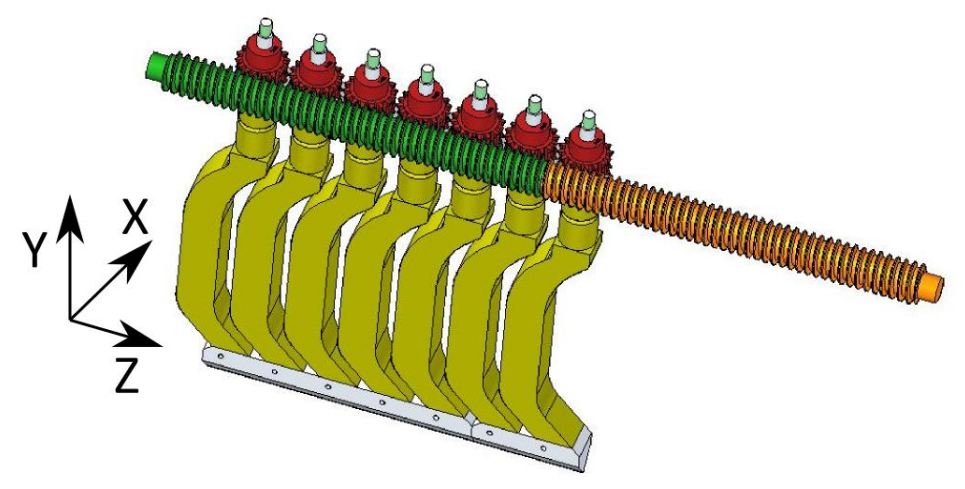

Figure 4. Exposed view of the drive system for the Leuven bend tooling system 
The ability to turn the segments separately allows for configuring tools with varying section over their length (Z-axis). This enables the bending of new variable profile geometries as depicted in Figure 5.
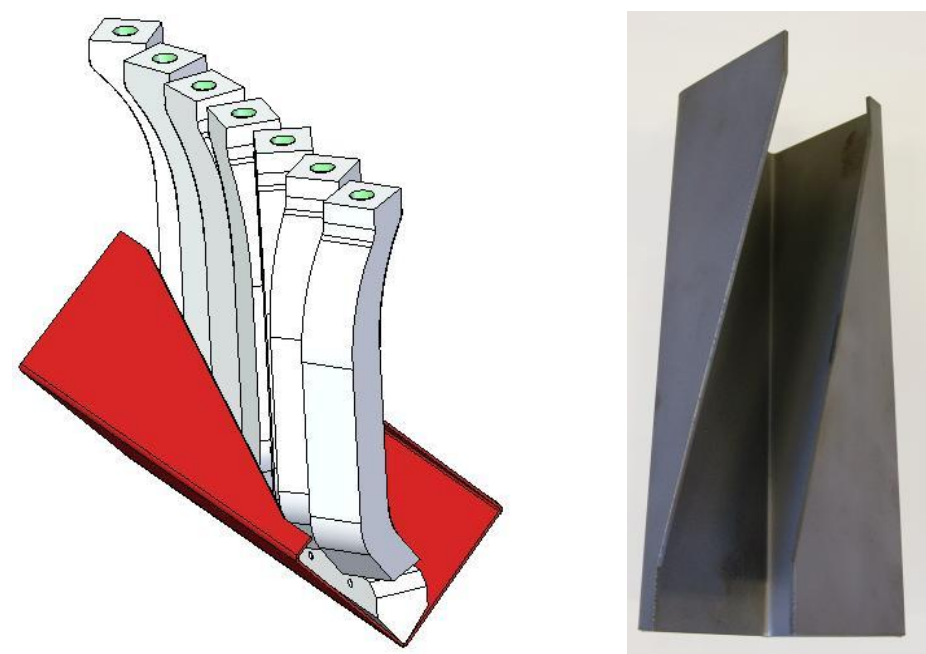

Figure 5. New variable profile geometry enabled by the adjustable tooling system

The upper and lower part of each C-segment has a cylindrical geometry at the top, which fits into the clamp, and a punch tip at the bottom. This allows for rotation of the C-segments. To eliminate clearance and align the punch correctly, the segments are clamped tightly at the top during bending. The clamping system aligns the punch and forces it to a straight position during the bending process. Figure 6 illustrates the full punch setup.

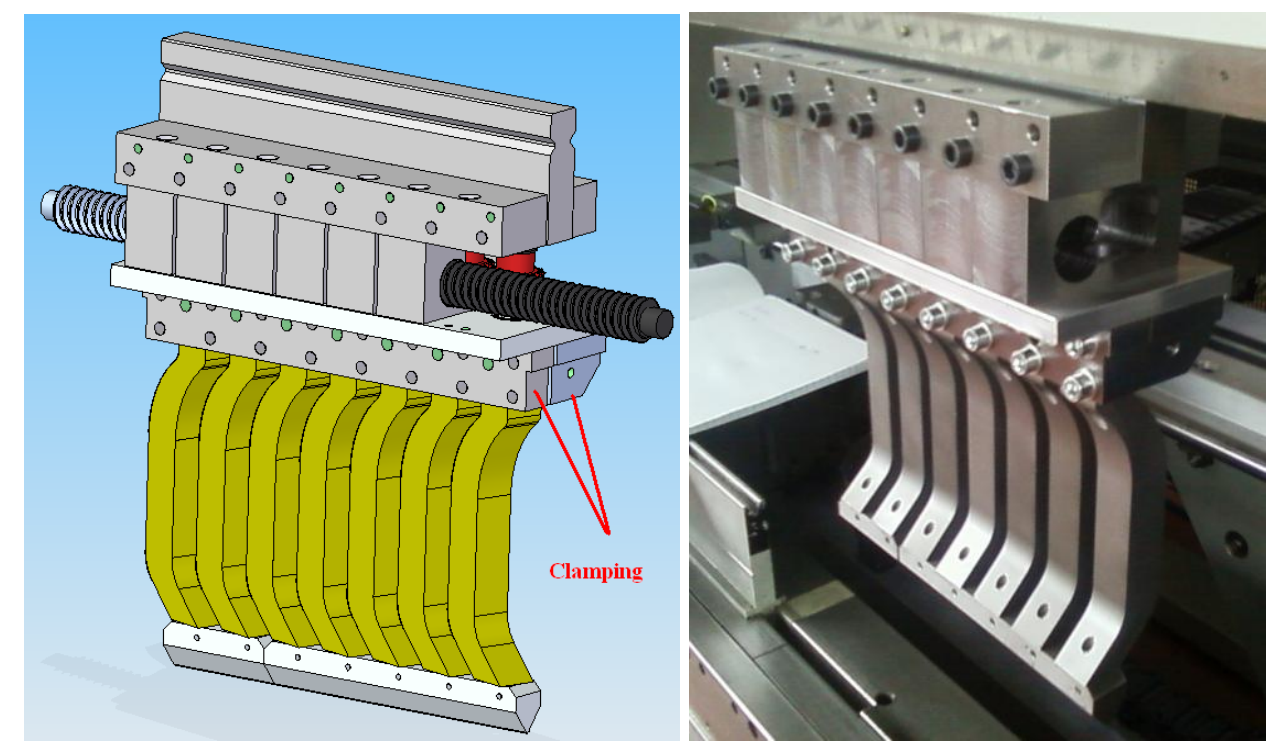

Figure 6. Punch assembly, shown for two punch members

More than one punch member, consisting of a clamping configuration, punch tip and multiple interconnecting C-segments, is needed when different specific bending lengths occur in parts to be manufactured. However, only a single tool system is required. This facilitates the possible use of an automatic tool changer. Using only a single set of controllable tool members, which can be shifted onto the press brake as depicted in Figure 7, different lengths of tooling can be made. 


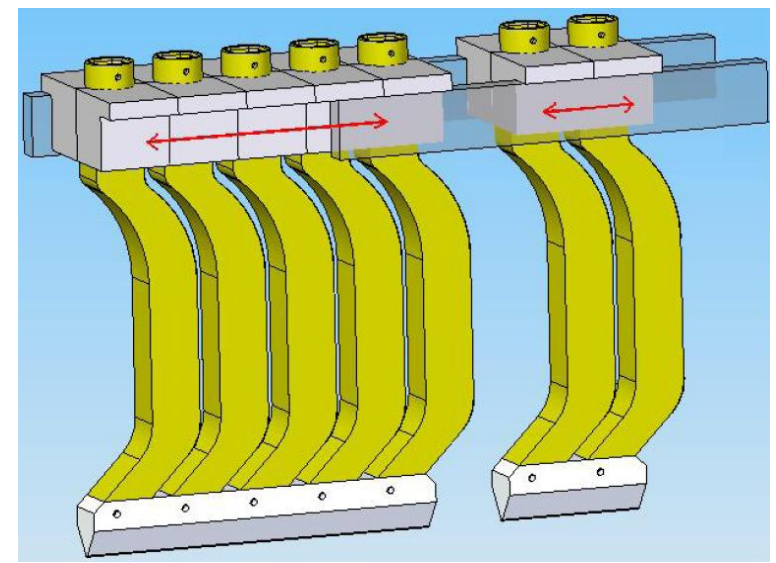

Figure 7. Concept of sliding extra segments to vary the bend length

\section{Control algorithm.}

An algorithm was developed to accurately predict the ability to bend a part with this tool as well as the optimal rotation angle for each $\mathrm{C}$-segment of the punch. For a given geometry the developed software solution evaluates each possible cross section of the tool and chooses the optimal one taking into account the part geometry and rotation time of the segments.

The calculation of a collision free segment orientation is performed using a $2 \mathrm{D}$ representation of the punch and part geometry. Figure 8 illustrates the side view of the punch bending a part containing three bend lines. The inner and outer contour line of the segments, depend on the rotational position of the segments. Changing the rotational position changes the inner and outer contour and allows for collision prevention.

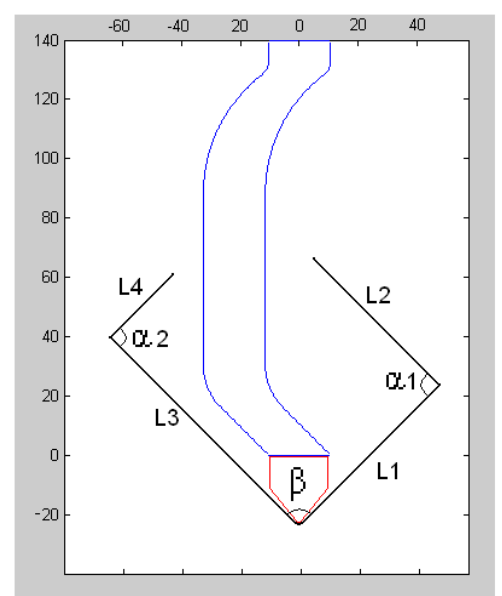

Figure $8.2 \mathrm{D}$ representation of the tool section for determining the optimal tool rotation

\section{Validation}

Structural validation. A prototype tool set was manufactured from UHB 11. The load bearing capacity was verified by bending sheets of DC 01 steel up to a thickness of $6 \mathrm{~mm}$ without permanent plastic deformation. This allowed verification of the predicted load bearing capacity as obtained by finite element simulations. Using high-strength tool steels would allow further increase in the achievable load capacity for the selected design configuration.

The stiffness of a punch greatly influences both the accuracy of the position of a bend line as well as the accuracy of the bend angle. Loaded by the forming forces, a punch will undergo a limited elastic deformation. To verify the magnitude of this deformation for the punch described in this paper, a set of bend tests was performed using a punch consisting of five $\mathrm{C}$-segments, which results in a bend 
length of $100 \mathrm{~mm}$. The punch was oriented in the different configurations and displacements were measured. A sheet of $1 \mathrm{~mm}$ DCO1 and a die with of $12 \mathrm{~mm}$ were used for all reported tests.

The deformation was measured by means of digital gauges with a measurement resolution of $2 \mu \mathrm{m}$, mounted on the punch parallel to the three principal axes of the press brake (see Fig. 9)

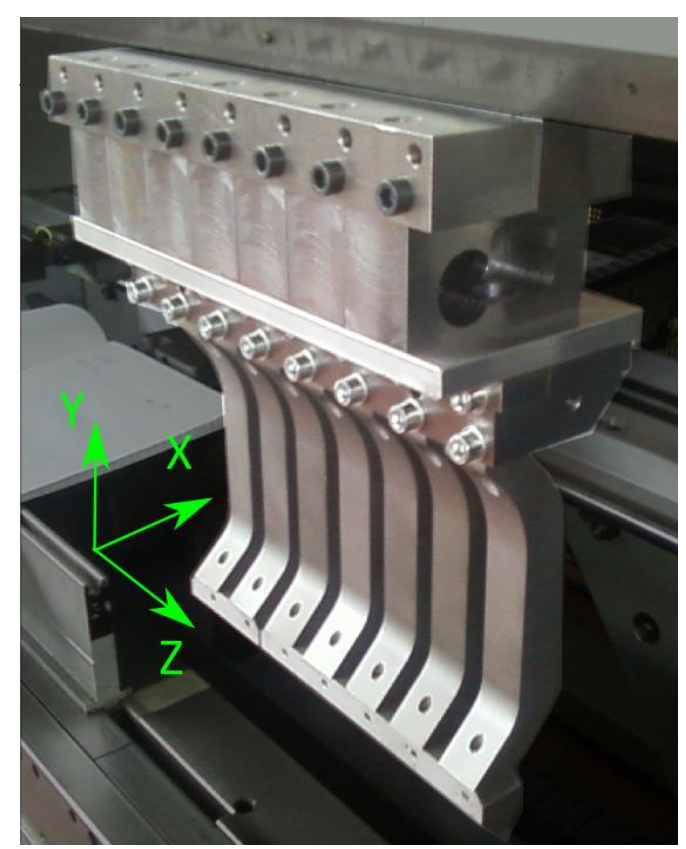

Figure 9. Orientation of distance gauges

Displacements in $\mathrm{Z}$ direction were only significant when the punch is oriented in left or right horn configuration. This is to be expected because in other configurations the tool is symmetric in $\mathrm{Z}$ direction. After averaging 5 test measurements, the results show a displacement of the punch tip of $0.026 \mathrm{~mm}$ in negative $\mathrm{Z}$ direction for the left horn configuration and a displacement of $0.027 \mathrm{~mm}$ in positive $\mathrm{Z}$ direction for the right horn configuration. The tests show a small spread of maximum $0.003 \mathrm{~mm}$ and the magnitude of the displacement between left and right horn configuration is comparable but in opposite direction.

A displacement in Y direction is expected to be consistent throughout all configurations of the tool. These displacements were measured both close to the left and right side of the tool for a left horn configuration to verify that the tool stays parallel to the die. The average result of 5 repeated measurements shows a displacement of $0.038 \mathrm{~mm}$ in the positive Y direction for the left side of the tool. The right side of the tool has a displacement of $0.035 \mathrm{~mm}$ in the positive $\mathrm{Y}$ direction. This means the tool stays parallel and has only a limited compression.

A displacement in $\mathrm{X}$ direction was measured for all configurations close to the left and right side of the tool to verify the orientation of the tool with respect to the die. The results are shown in Table1.

Table1. Displacement of the punch tip in X direction during bending

\begin{tabular}{|l|c|c|}
\cline { 2 - 3 } \multicolumn{1}{c|}{} & $X_{\text {left }}$ & $X_{\text {right }}$ \\
\cline { 2 - 3 } \multicolumn{1}{c|}{} & {$[\mathrm{mm}]$} & {$[\mathrm{mm}]$} \\
\hline horn left & 0.066 & 0.097 \\
\hline horn right & 0.056 & 0.079 \\
\hline $\begin{array}{l}\text { gooseneck } \\
\text { front }\end{array}$ & 0.083 & 0.100 \\
\hline $\begin{array}{l}\text { gooseneck } \\
\text { back }\end{array}$ & 0.032 & 0.032 \\
\hline
\end{tabular}


The results of these tests are relatively high. This can be explained by the limited accuracy of the prototype. In unloaded condition the punch tip showed a deviation of $0.1 \mathrm{~mm}$ with respect to the expected position in the center of the die. While loaded under the high forming forces the punch tip is pushed towards the center of the die and thus has a displacement in a range up to $0.1 \mathrm{~mm}$

\section{Conclusions}

Through the use of a modular, rotatable punch tool for press brakes, many tool changes can be avoided. The tool presented in this paper can be adapted to different standard punch profiles, such as straight tools, gooseneck tools and horn tools. This tool system can be automated to quickly reconfigure itself between bend actions without the need for labor intensive setup. When equipped with a robot and an automatic material handling system, this enables the press brake to run for a long period, producing a wide variety of parts without human interference.

Finally the tool system was validated structurally by means of a range of bending tests, confirming its load bearing capacity and documenting its stiffness.

\section{References}

[1] Y. Vanhecke: Work study and time analysis for sheet metal production (Master thesis Katholieke Universiteit Leuven), (2006)

[2] J. Gheysens and P. Vansteenwegen: Bewegingsanalyse en tijdsraming voor buigprocessen (Master thesis Katholieke Universiteit Leuven), (2002)

[3] D. Cattrysse, P. Beullens, P. Collin, J.R. Duflou and D. Van Oudheusden: International Journal of Production Research, 44:20, 4311-4327, (2006)

[4] U. Alva and S.K. Gupta: Robotics and Computer Integrated Manufacturing 17 (2001) 33-47

[5] T.H.M. Nguyen: 2005, Automatic tool selection and dimensional accuracy verification in computer aided process planning for sheet metal bending (PHD dissertation Katholieke Universiteit Leuven), (2005)

[6] B. Verlinden: Cost estimation and integrated production planning for sheet metal laser cutting and air bending ( PHD dissertation Katholieke Universiteit Leuven), (2008)

[7] J.R. Duflou: Automatic design verification and process planning for bent sheet metal parts (PHD dissertation Katholieke Universiteit Leuven), (1999) 